\title{
Exploring Biochemical Pathways for Mono-Ethylene Glycol (MEG) Synthesis from Synthesis Gas
}

\author{
M. Ahsanul Islam ${ }^{1}$, Noushin Hadadi ${ }^{2}$, Meric Ataman ${ }^{2}$, Vassily Hatzimanikatis ${ }^{2, *}$, and Gregory \\ Stephanopoulos ${ }^{1, *}$
}

${ }^{1}$ Department of Chemical Engineering, Massachusetts Institute of Technology, Cambridge, MA 02139, United States

${ }^{2}$ Institute of Chemical Sciences and Engineering, Ecole Polytechnique Fédérale de Lausanne, CH-1015, Lausanne, Switzerland

*Corresponding authors: gregstep@ mit.edu, vassily.hatzimanikatis@epfl.ch 


\begin{abstract}
Mono-ethylene glycol (MEG) is an important petrochemical with widespread use in numerous consumer products. The current industrial MEG-production process relies on non-renewable fossil fuel-based feedstocks, such as petroleum, natural gas, and naphtha; hence, it is useful to explore alternative routes of MEG-synthesis from gases as they might provide a greener and more sustainable alternative to the current production methods. Technologies of synthetic biology and metabolic engineering of microorganisms can be deployed for the expression of new biochemical pathways for MEG-synthesis from gases, provided that such promising alternative routes are first identified. We used the BNICE.ch algorithm to develop novel and previously unknown biological pathways to MEG from synthesis gas by leveraging the Wood-Ljungdahl pathway of carbon fixation of acetogenic bacteria. We developed a set of useful pathway pruning and analysis criteria to systematically assess thousands of pathways generated by BNICE.ch. Published genome-scale models of Moorella thermoacetica and Clostridium ljungdahlii were used to perform the pathway yield calculations and in-depth analyses of seven (7) newly developed biological MEG-producing pathways from gases, including $\mathrm{CO}_{2}, \mathrm{CO}$, and $\mathrm{H}_{2}$. These analyses helped identify not only better candidate pathways, but also superior chassis organisms that can be used for metabolic engineering of the candidate pathways. The pathway generation, pruning, and detailed analysis procedures described in this study can also be used to develop biochemical pathways for other commodity chemicals from gaseous substrates.
\end{abstract}




\section{Introduction}

Commodity chemicals such as Ethylene glycol or Mono-ethylene glycol (MEG; 1,2-Ethanediol) and its polymers are the building blocks of modern industrial society due to their widespread use in myriad consumer products. MEG is ubiquitously used in automobile radiators as an antifreeze agent, in the production of polyester fibers, films, and Polyethylene terephthalate (PET) resins for plastic bottles (Forkner et al., 2000; Rebsdat and Mayer, 2000; ShellFactsSheet, 2016). Because of its extensive applications in the manufacturing of consumer goods, the global market volume of MEG was 17 million tons per year in 2013 and projected to grow to 23 million tons per year by 2020 at a market value of US\$ 33.36 billion (GrandViewResearchReport, 2016; ShellFactsSheet, 2016). However, the present industrial process of MEG-production from Ethylene oxide hydrolysis completely depends on non-renewable fossil fuel-based feedstocks, such as petroleum, natural gas, and naphtha to obtain Ethylene by thermal cracking (Forkner et al., 2000; Rebsdat and Mayer, 2000). As such, this process contributes to the release of harmful greenhouse gases, including Carbon dioxide $\left(\mathrm{CO}_{2}\right)$ and Methane $\left(\mathrm{CH}_{4}\right)$ to the atmosphere. Furthermore, the industrial process also suffers from low selectivity of MEG because higher order glycols are also produced in the process due to the increased reactivity of Ethylene oxide towards MEG than to water (Forkner et al., 2000; Rebsdat and Mayer, 2000). It is thus of interest to explore the routes for biological production of MEG from renewable feedstocks such as sugars using engineered microbial organisms, and this interest has obtained significant attention from the scientific community recently (Alkim et al., 2015; Liu et al., 2013; Pereira et al., 2016a; Pereira et al., 2016b; Stephanopoulos et al., 2013). 
So far, bio-based production of MEG using engineered Escherichia coli strains has been reported from pentose and hexose sugars, including xylose (Alkim et al., 2015; Liu et al., 2013; Pereira et al., 2016a), arabinose (Pereira et al., 2016a), and glucose (Pereira et al., 2016b). These processes are very promising since the sugars are abundant in lignocellulosic biomass feedstocks obtained from agricultural wastes, forestry residues, grasses, and woody materials (Anwar et al., 2014; Menon and Rao, 2012). Another alternative and useful feedstock could be gaseous substrates, such as $\mathrm{CO}_{2}, \mathrm{CO}$, and $\mathrm{H}_{2}$, provided that biochemical pathways for converting such gases into MEG can be identified. There is no shortage of the greenhouse gas, $\mathrm{CO}_{2}$, in the atmosphere, and $\mathrm{CO}$ and $\mathrm{H}_{2}$ are components of synthesis gas (syngas), which can be cheaply and abundantly obtained from natural gas, coal gasification, gasification of plant biomass, and pyrolysis of organic waste products (Mackaluso, 2007; Rostrup-Nielsen and Christiansen, 2011). Thus, the biological conversion of these gases into MEG could provide an attractive alternative to both greenhouse gas emissions and the unsustainable, fossil fuel-based MEG-production process.

The design/identification of biochemical pathways to convert gases to MEG is non-trivial (Medema et al., 2012) and can be tackled with the help of state-of-the-art cheminformatics and metabolic engineering tools, such as BNICE.ch (Hadadi et al., 2016; Hatzimanikatis et al., 2005), DESHARKY (Rodrigo et al., 2008), GEM-path (Campodonico et al., 2014), RetroPath (Fehér et al., 2014), and XTMS (Carbonell et al., 2014). Almost all of these cheminformatics tools are based on the retrobiosynthesis principle (Carbonell et al., 2013; Hadadi and Hatzimanikatis, 2015; Medema et al., 2012), in which a set of defined biotransformation rules are iteratively applied to connect a target compound "retrosynthetically" to the metabolites of either a host organism's metabolic network, or a biochemical database such as KEGG (Kanehisa et al., 2016), 
MetaCyc (Caspi et al., 2014), and PubChem (Kim et al., 2016). Although these cheminformatics tools are capable of generating both novel and biologically well-known biotransformations, almost all of them suffers from the combinatorial explosion of pathway generation resulting in hundreds and thousands of generated pathways; thereby, posing an important challenge of postprocessing of the pathways to decipher and select useful and meaningful ones from the redundant pathways.

In this study, we applied the BNICE.ch framework and algorithm (Hadadi et al., 2016; Hatzimanikatis et al., 2005; Li et al., 2004) to develop biochemical pathways for converting syngas $\left(\mathrm{CO}+\mathrm{H}_{2}\right)$ to generate MEG. Since $\mathrm{CO}_{2}, \mathrm{CO}$, and $\mathrm{H}_{2}$ gases can be efficiently converted to Acetyl-CoA by the Wood-Ljungdahl pathway (WLP) of carbon fixation (Fast and Papoutsakis, 2012; Fuchs, 2011), we employed BNICE.ch to design/identify biochemical pathways connecting Acetyl-CoA to MEG. Then, we developed and implemented a set of pathway pruning and analysis criteria for post-processing of the large number of generated pathways by BNICE.ch. Because this algorithm can generate both novel i.e., biologically unknown reactions and biologically known reactions i.e., reactions present in a biological database such as KEGG (Kanehisa et al., 2016), further pathway screening was conducted to identify only KEGGreactions containing pathways to generate MEG. Afterwards, these MEG-producing pathways were analyzed in detail using Moorella thermoacetica and Clostridium ljungdahlii as the host microbes because these anaerobic acetogenic bacteria use WLP to produce Acetyl-CoA from $\mathrm{CO}_{2}, \mathrm{CO}$, and $\mathrm{H}_{2}$ (Drake, 1994; Drake et al., 2008; Kopke et al., 2010; Pierce et al., 2008). The pathways were further analyzed to satisfy thermodynamic feasibility and to ensure that the pathway genes were available either in the genomes of the host organisms, or in other sequenced 
genomes in biological databases. We finally used the published genome-scale models of $M$. thermoacetica (Islam et al., 2015) and C. ljungdahlii (Nagarajan et al., 2013) to estimate the pathway yields, as well as the effect of metabolic diversity of the host microbes on the estimated pathway yields of MEG-producing pathways. Such detailed analysis helped identify a better host for metabolic engineering of the designed MEG-producing pathways from syngas.

\section{Materials and methods}

\subsection{Generation of the mono-ethylene glycol (MEG)-producing pathways}

We generated a reconstructed biochemical reaction network (Tables S1 and S2 in the supplementary data) for MEG using the BNICE.ch algorithm (Hadadi and Hatzimanikatis, 2015; Hatzimanikatis et al., 2005; Li et al., 2004). The algorithm, as well as its functionality and applications have been described in details elsewhere (Finley et al., 2009; Hadadi et al., 2016; Hatzimanikatis et al., 2005; Li et al., 2004). Briefly, BNICE.ch requires a number of inputs to generate a biochemical reaction network leading to the target compound (Hadadi et al., 2016). These inputs include a set of defined generalized reaction rules/reaction operators ( $\mathrm{Li}$ et al., 2004) derived from the $3^{\text {rd }}$ level information of the Enzyme Commission (EC) classification

system recommended by the Nomenclature Committee of the International Union of Biochemistry and Molecular Biology (NC-IUBMB, 1992); a reference database such as KEGG (Kanehisa et al., 2016); and cofactor and starting compound(s) such as NAD, NADP, and MEG, representing the $4^{\text {th }}$ level of the EC classification system information (i.e., reactants and substrates). BNICE.ch then retrosynthetically generated a large biochemical network of all 
possible reactions connecting MEG to the metabolites of KEGG by iteratively applying 722 generalized reaction rules (Hadadi et al., 2016). Successively, a graph-based pathway search algorithm implemented in the BNICE.ch framework (Hatzimanikatis et al., 2005) was used to generate all possible pathways connecting MEG to Acetyl-coA using up to 4 steps/reactions (Table S3 in the supplementary data). The Gibbs free energy of reactions for all pathways was estimated using the group contribution method (Jankowski et al., 2008).

\subsection{Curation of the mono-ethylene glycol (MEG)-producing pathways}

The BNICE.ch generated pathways were curated based on the following pathway pruning criteria: (1) number of reactions/steps involved in a pathway i.e., if a pathway contains 2,3 , or 4 reactions; (2) presence of common intermediate metabolites in the last step of a pathway i.e., if the same metabolite pairs are included in the last reaction/step of multiple pathways, then the pathways are categorized based on that information; (3) presence of Oxygen as a reactant in a pathway reaction/step i.e., if a pathway includes oxygen-containing reaction(s), that pathway is excluded from further analysis; and (4) presence of external metabolites-requiring reaction in a pathway i.e., if a pathway contains reactions that require metabolites not produced by the native metabolism of the host organisms, that pathway is not included in subsequent analysis. More detailed description of these criteria, the rationale behind their selection, and their implementation to pruning the BNICE.ch generated pathways are elaborated in the results and discussion section. The pruned pathways are then analyzed using the pathway search algorithm to remove novel i.e., biologically unknown reactions/steps not present in the KEGG database. After identifying all pathways containing only KEGG-reactions, the pathways are further 
assessed on the basis of these criteria: (1) the overall thermodynamic feasibility of a pathway, (2) the number of reactions/genes present in a pathway that are native to the host organisms, (3) the number of genes/reactions present in a pathway that are found in other sequenced genomes in biological databases, and (4) the estimated pathway yield. Further analysis and implementation of these criteria are discussed in results and discussion.

\subsection{Estimation of pathway yields of the mono-ethylene glycol (MEG)-producing pathways}

The pathway yields of all pruned and accepted MEG-producing pathways, as well as the pathways containing only KEGG-reactions, or biologically known reactions/steps were estimated using the published genome-scale metabolic models of Moorella thermoacetica (Islam et al., 2015) and Clostridium ljungdahlii (Nagarajan et al., 2013). Flux balance analysis (FBA) (Orth et al., 2010) was used for maximizing the MEG-production objective function in the $M$. thermoacetica and C. ljungdahlii models. The COBRA toolbox (Becker et al., 2007; Hyduke et al., 2011) was used for FBA-implementation, and all model simulations were conducted in the MATLAB environment (the MathWorks Inc., Natick, MA) using the IBM ILOG's CPLEX 12.5.1 optimization solver.

\section{Results and discussion}

\subsection{Enumeration of the Acetyl-CoA to mono-ethylene glycol (MEG)-producing pathways}

The implementation of BNICE.ch algorithm using a total of 722 generalized reaction rules (Hadadi et al., 2016) and MEG as a starting compound retrosynthetically generated a network of 
18,289 reactions and 2,283 metabolites after converging at 28 iterations (Figure 1, and Tables S1 and S2 in the supplementary data), meaning that no new metabolite and no new reaction can be generated from further runs of the algorithm. Of the total 18,289 reactions in the network, only $18 \%$ or 3,240 are found in the KEGG database (Kanehisa et al., 2016) while the rest (15,049 or $82 \%$ ) are novel reactions without having any existence in KEGG with a KEGG-reaction number (Figure 1). Since we used KEGG as the reference database and input to BNICE.ch, all compounds/metabolites in the generated network belonged to the KEGG-compounds. The graphbased pathway search algorithm in BNICE.ch was then applied to generate 1, 100, and 2,091 pathways of 2, 3, and 4-reaction/step lengths, respectively, connecting Acetyl-CoA to MEG (Table S3 in the supplementary data). Interestingly, no 1-step pathway(s) connecting AcetylCoA to MEG was identified although the algorithm generated more than 2,100 pathways in total (Table S3 in the supplementary data).

Due to the generation of a large number of MEG-producing pathways, we resorted to a set of pathway pruning criteria (Materials and methods) to curate them for further analyses. These criteria were established by analyzing the pathway length and structure, types of reactants involved in a pathway reaction/step, and the nature of the host microbes' metabolic capability. The first pruning criterion - number of reactions/steps involved in a pathway (Materials and methods) — was based on the pathway length, and we opted to generate only up to 4-step long pathways due to combinatorial explosion of the number of generated pathways with the increase in pathway length. After careful observation of all pathways, we also noticed some similarities in the last step of both 3 and 4-step pathways with respect to reactants/metabolites involved in the final step. This observation led to the emergence of the second pruning criterion: presence of 
common intermediate metabolites in the last step of a pathway (Materials and methods), and its implementation (Figure 2) resulted in four (Figure 2A) and six (Figure 2B) categories of 3 and 4step long pathways, respectively. The categories were named according to the metabolite pairs involved in the last step of a pathway. Thus, Ethanol-MEG category included only those 3 and 4step pathways (56 and 812 in total in Figures $2 \mathrm{~A}$ and 2B, and in Tables S4, S8 in the supplementary data) containing the conversion of Ethanol to MEG in the last step. Similarly, Glycerate-MEG, Glycolaldehyde-MEG, Ethylene-MEG, Ethylene oxide-MEG, and Propylene glycol-MEG categories of pathways were identified, and included 6, 36, 2 and 128, 987, 16, 4, 144 number of 3 and 4-step pathways, respectively (Tables S5 - S7 and S9 - S13 in the supplementary data). The Ethylene oxide-MEG and Propylene glycol-MEG categories of pathways (4 and 144 in total) were found only for the 4-step long pathways (Figure 2B and Tables S12, S13 in the supplementary data).

The third pruning criterion, presence of Oxygen as a reactant in a pathway step (Materials and methods), confirmed that only anaerobic pathways were selected due to choosing anaerobic acetogenic bacteria - M. thermoacetica and C. ljungdahlii - as chassis organisms for further analyses of the BNICE.ch generated pathways. Finally, the fourth pathway-pruning criterion (Materials and methods) stemmed from the observation that many of the generated pathways involved reactions requiring the supply of metabolites not naturally produced by the host cell metabolism. For instance, Acrylate and Acetol are involved in some 3 and 4-step pathways (Tables S1 and S2 in the supplementary data), but these metabolites are not produced by the wild type $M$. thermoacetica and $C$. ljungdahlii; hence, pathways involving such external metaboliterequiring reactions were pruned and omitted from further analyses using the fourth criterion: 
presence of external metabolites-requiring reaction in a pathway. The third and fourth pruning criteria were then separately applied to the different categories of 3 and 4-step pathways (Figures $2 \mathrm{~A}$ and 2B), yielding a total of 23 three-step (Figure 2A and Tables S14 - S16 in the supplementary data) and 209 four-step (Figure 2B and Tables S17 - S19 in the supplementary data) long accepted pathways from more than 2,100 pathways generated by BNICE.ch. Notably, no 3 or 4-step pathway from Ethylene-MEG, Ethylene oxide-MEG, and Propylene glycol-MEG categories passed the third and fourth pruning criteria due to the requirement of either Oxygen, Acetaldehyde, or Ethylene (non-native metabolites of M. thermoacetica and C. ljungdahlii) as reactant(s) in the pathway reaction(s).

Both reactions/steps of the only 2-step long MEG-producing pathway (Table S3 in the supplementary data) belonged to KEGG, but the $1^{\text {st }}$ reaction (R05351) is an orphan enzyme, i.e., the gene information for the reaction is unknown (Shearer et al., 2014; Sorokina et al., 2014; Watschinger and Werner, 2013). A closer look at the accepted 3 and 4-step pathways (Figure 3) revealed that at least one novel reaction existed in each pathway, while no pathway was found in which all reactions/steps were known to be biological (Figures 3A and 3B), i.e., belonged to the KEGG database with a KEGG-reaction number. Thus, in spite of generating more than 2,100 pathways with BNICE.ch, no 3 or 4-step pathway containing all KEGG-reactions was identified. It is noteworthy to mention that the Ethanol-MEG (4 two-KEGG-step pathways in Figure 3A and 12 three-KEGG-step pathways in Figure 3B) and Glycolaldehyde-MEG (2 two-KEGG-step pathways in Figure 3A and 12 three-KEGG-step pathways in Figure 3B) categories included pathways containing more KEGG-steps than those in the Glycerate-MEG category (0 twoKEGG-step pathways in Figure 3A and 0 three-KEGG-step pathways in Figure 3B); this 
indicates that both Ethanol and Glycolaldehyde are closer to MEG than Glycerate in the biochemical network generated by BNICE.ch, which is consistent with the current knowledge, (e.g., in KEGG).

\subsection{Analyses of the reconstructed biochemical networks and pathway yields of the accepted pathways}

In order to obtain a clear topological view of the connectivity of metabolites/reactants, as well as the number of KEGG-reactions involved in different pathways, the reconstructed biochemical networks of pruned and accepted pathways connecting Acetyl-CoA to MEG are generated and depicted in Figure 4. The networks of 3-step pathways (Figures 4A, 4B, 4C) are simpler than 4step pathways (Figures 4D, 4E, and 4F) with the latter networks being more densely connected than the former ones. Among the 3-step pathways, the Ethanol-MEG (Figure 4A) and Glycolaldehyde-MEG (Figure 4C) categories included pathways with two KEGG-reactions, while only one KEGG-reaction was identified for the pathways in the Glycerate-MEG category (Figure 4B). Inspection of the densely connected networks of 4-step pathways showed that the $2^{\text {nd }}$ and $3^{\text {rd }}$ steps/reactions have more variations than the $1^{\text {st }}$ and $4^{\text {th }}$ steps in a pathway (Figures $4 \mathrm{D}, 4 \mathrm{E}$, and $4 \mathrm{~F})$, and this flexibility in the intermediate steps actually helped to generate a large number of 4-step pathways $(2,091)$ as compared to only 100 three-step pathways. Interestingly, this flexibility is more visible for the 4-step pathways in the Ethanol-MEG and Glycerate-MEG categories than in the Glycolaldehyde-MEG category due to the presence of highly connected acetaldehyde (Figure 4D) and pyruvate (Figure 4E) metabolites in the biochemical networks of pathways included in those categories. 
In order to assess the compatibility of the accepted pathways with the host microbes' metabolism, we also estimated the MEG-yields of all the 3 and 4-step long accepted pathways (Figure 5 and Tables S14 - S19 in the supplementary data). The pathway yields were estimated using the published genome-scale models of M. thermoacetica (Islam et al., 2015) (Figures 5A and 5B) and C. ljungdahlii (Nagarajan et al., 2013) (Figures 5C and 5D), and the substrates used for these yield estimations included Carbon dioxide $\left(\mathrm{CO}_{2}\right)$ and Hydrogen $\left(\mathrm{H}_{2}\right)$ (Figures 5A and 5C), and Carbon monoxide ( $\mathrm{CO}$ ) (Figures 5B and 5D) - the components of syngas and gaseous substrates of both host bacteria. The theoretical yield of MEG on the gaseous substrates (Table S20 in the supplementary data) was calculated using the concept of "degree of reductance" of an organic compound (Blanch and Clark, 1997), which essentially takes into account the reducing power of substrates per carbon atom basis to produce a product (Dugar and Stephanopoulos, 2011). The majority of the pathways produced MEG-yields similar to the theoretical MEG-yields of $0.74 \mathrm{~g} \mathrm{MEG} / \mathrm{g} \mathrm{H}_{2}+\mathrm{CO}_{2}$ and $0.44 \mathrm{~g} \mathrm{MEG/g} \mathrm{CO}$ for both hosts, but the compatibility of the pathways was different as observed from the MEG-yields with the C. ljungdahlii model (Figures 5C and 5D). In total, 33 pathways were not able to produce MEG with the $C$. ljungdahlii model hence resulted zero MEG-yields (Figure 5C and 5D), because one of the byproducts of these pathways, $\mathrm{H}_{2} \mathrm{O}_{2}$, was not consumed by any other reactions in the model; thus, rendering the pathways incompatible for the C. ljungdahlii metabolism. However, all 3 and 4-step pathways were compatible with the $M$. thermoacetica metabolism and produced MEG using the host's metabolic model (Figures 5A and 5B). 


\subsection{Generation of the pathways containing only KEGG-reactions}

Pathways converting Acetyl-CoA to MEG with novel reactions are exciting candidates for hypotheses generation and testing about candidate enzymes, but not for immediate implementation into host organisms via metabolic engineering. Although the pathway yields of the majority of the accepted pathways were similar to the theoretical MEG-yields on gaseous substrates with the host organisms' metabolic model (Figure 5), all of them included either orphan enzymes or novel reactions for which no gene/protein information is available. To this end, pathways containing only biologically known reactions i.e., KEEG-reactions are required to be identified. The biochemical networks of 3-step pathways (Figures 4A, 4B, and 4C) show that the $1^{\text {st }}$ reaction in Ethanol-MEG, Glycerate-MEG, and Glycolaldehyde-MEG categories is known, while the $2^{\text {nd }}$ reaction is known only for the pathways in the Ethanol-MEG category (Figure $4 \mathrm{~A}$ ) and the $3^{\text {rd }}$ reaction is a KEGG-reaction for the pathways belonging to the Glycolaldehyde-MEG category only (Figure 4C). Thus, to generate pathways with all KEGGreactions, we used the pathway search algorithm in BNICE.ch to connect Ethanol to MEG or Glycerate to MEG using up to 5-step long pathways. After analyzing the pathways with the developed pathway pruning criteria (Materials and methods), no pathway with all KEGGreactions was identified. We, then, opted to connect either Ethanol to Glycolaldehyde, or

Glycerate to Glycolaldehyde with up to 5-step pathways, so that the biologically known steps of Acetyl-CoA to Ethanol or Glycerate and Glycolaldehyde to MEG can be combined to generate pathways from Acetyl-CoA to MEG containing only KEGG-reactions. 
BNICE.ch generated 189,513 and 71,880 pathways of up to 5-step length connecting Glycerate to Glycolaldehyde and Ethanol to Glycolaldehyde, respectively. We analyzed the pathways using the same pathway pruning criteria (Materials and methods), as well as to search for pathways containing only KEGG-reactions. This analysis (Figure 6) resulted in the identification of 4 pathways connecting Glycerate to Glycoladehyde using 2, 3, 4, and 6 reactions/steps (Figure 6A), and 3 pathways of 3 and 5-step lengths connecting Ethanol to Glycolaldehyde (Figure 6B). After combining these pathways with the biologically known KEGG-reaction of converting Glycolaldehyde to MEG (R01781), we obtained 7 pathways in total to convert Glycerate and Ethanol to MEG. The Glycerate to MEG pathways are denoted as MEG1, MEG2, MEG3, and MEG4 (Figure 6A) while MEG5, MEG6, and MEG7 represent pathways converting Ethanol to MEG (Figure 6B). Thus the overall pathways converting syngas to MEG (Figure 6C) can be described as a combination of the Wood-Ljungdahl pathway converting syngas to Acetyl-CoA, conversion of Acetyl-CoA to either Glycerate by gluconeogenesis or Ethanol by fermentation, and ultimately, the conversion of Glycerate or Ethanol to MEG via Glycolaldehyde formation by the designed/engineered pathways (Figure 6C).

\subsection{Thermodynamic feasibility, gene availability, and pathway yield analysis of the mono- ethylene glycol (MEG)-producing pathways with only KEGG-reactions}

The 7 pathways containing only KEGG-reactions to convert Glycerate or Ethanol to MEG were further evaluated (Figures 7 and 8) on the basis of overall pathway Gibbs energy change, gene availability based on the number of host organisms' genes, or genes from a sequenced genome encoding the required enzymes present in a pathway, and the estimated pathway yield. Using 
these criteria, the pathways were also compared with the published biosynthetic pathways of MEG-production from xylose (Liu et al., 2013) and glucose (Pereira et al., 2016b).

In order to estimate the overall pathway Gibbs energy change, the standard Gibbs energy of formation of the compounds participating in a reaction was first calculated using the group contribution method (Jankowski et al., 2008). Then the standard reaction Gibbs energy of each reaction/step in a pathway was calculated, and the overall pathway Gibbs energy change was determined by adding up the reaction Gibbs energies of all reactions included in the pathway. This analysis is especially useful for estimating the overall thermodynamic feasibility of the pathways containing both endergonic or non-spontaneous (i.e., positive reaction Gibbs energy change) and exergonic or spontaneous (i.e., negative reaction Gibbs energy change) reactions. For instance, both MEG5 and MEG6 pathways have exergonic and endergonic steps, but MEG5 is thermodynamically more favorable than MEG6 because the overall pathway Gibbs energy change of MEG5 is negative while MEG6 is positive (Figure 7A). Another notable feature illustrated by this analysis is that the shorter length pathways have more negative pathway Gibbs energy changes than the longer length pathways. In total, 6 pathways including the published biosynthetic pathways (Figure 7A) showed an overall negative pathway Gibbs energy change suggesting their superior thermodynamic feasibility as compared to the remaining 3 pathways (MEG2, MEG6, and MEG7).

Next, the gene availability was assessed for the 9 MEG-producing pathways based on whether the genes associated with the candidate pathways are found either in the host microbes (i.e., $M$. thermoacetica and C. ljungdahlii), or in other sequenced genomes in KEGG (Figure 7B). Except 
of MEG3 and the two published pathways, the rest of the analyzed pathways have at least one reaction/step associated with gene(s) found in the M. thermoacetica and C. ljungdahlii genomes; only MEG7 contains 2 reactions with $M$. thermoacetica and $C$. ljungdahlii genes (Figure 7B). However, most of the genes associated with the reactions of all pathways are not found in $M$. thermoacetica and C. ljungdahlii genomes, but in other sequenced genomes. Information about these genes can be easily obtained from KEGG database using the identified KEGG-reaction number of the steps involved in a pathway (Figure 7B). Notably, MEG6 includes 2 steps for which the KEGG reaction number is known, but the gene information is unknown because they are orphan enzymes (Shearer et al., 2014; Sorokina et al., 2014; Watschinger and Werner, 2013). Overall, both MEG1 and MEG7 pathways have similar portion (33\%) of the host microbes' gene-containing reactions, but MEG1 is a better candidate for metabolic pathway engineering purposes in hosts $M$. thermoacetica and $C$. ljungdahlii due to having a negative overall pathway Gibbs energy change (Figure 7A).

Finally, we examined the pathway yields of the 9 MEG-producing pathways containing only KEGG-reactions using the published genome-scale models of hosts $M$. thermoacetica (Islam et al., 2015) and C. ljungdahlii (Nagarajan et al., 2013) as before (Figure 8 and Table S20 in the supplementary data). The pathway yields were estimated for the syngas components and gaseous substrates of the host microbes, including Carbon dioxide $\left(\mathrm{CO}_{2}\right)$ and Hydrogen $\left(\mathrm{H}_{2}\right)$ (Figure 8A), and Carbon monoxide (CO) (Figure 8B). All of the 7 newly designed pathways, as well as the 2 published pathways are completely compatible with both hosts as evident from the resulting MEG-yields using those pathways in the models. Notably, not all MEG-producing pathways were able to achieve the calculated theoretical MEG-yields on the gaseous substrates, even 
though the model simulations were conducted for the maximization of MEG-production objective function. For instance, only MEG2, MEG5, and MEG7 resulted in MEG-yields similar

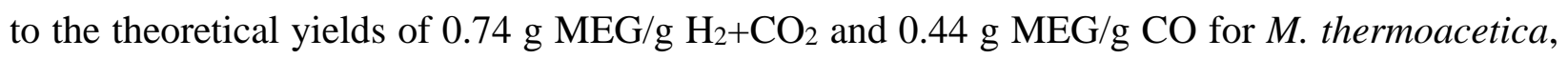
and only MEG5 and MEG7 produced the theoretical MEG-yields for C. ljungdahlii (Figures 8A and $8 \mathrm{~B}$ ). Also, the same pathways yielded different MEG on different substrates as evident from the estimated yields of MEG1, MEG3, MEG4, MEG6, and the published MEG-producing pathways (Liu et al., 2013; Pereira et al., 2016b).

The difference in MEG-yields of the pathways can be attributed to both pathway structure and metabolic diversity of the host microbes. The 4 MEG-producing pathways comprising the conversion of Glycerate to MEG (MEG1 - MEG4 in Figures 8A and 8B) yielded less MEG than the pathways that included the Ethanol to MEG conversion steps (MEG5 - MEG7 in Figures 8A and $8 \mathrm{~B}$ ); loss of a carbon atom to produce the two carbon compound MEG from the three carbon containing Glycerate using MEG1 - MEG4 pathways can contribute to this yield reduction. However, the yield of MEG2 is the highest among the Glycerate to MEG producing pathways because MEG2 includes a step (R00013 in Figure 6A), where one mole of $\mathrm{CO}_{2}$ is combined with Tartronate semialdehyde to produce two moles of Glyoxylate by a flavoprotein, Glyoxylate carboligase (Gupta and Vennesland, 1964). Thus, this pathway fixes one mole of carbon instead of losing one to produce MEG from Glycerate. Notably, the pathway yields estimated using the M. thermoacetica metabolic model were higher than that estimated using the C. ljungdahlii model. This yield difference can be attributed to the difference in autotrophic energy metabolism of these two host bacteria. Although the exact mechanism of their energy conservation during autotrophy still remains unresolved (Huang et al., 2012; Kopke et al., 2010; Mock et al., 2014; 
Schuchmann and Muller, 2014; Wang et al., 2013), modeling studies suggest that the autotrophic metabolism of M. thermoacetica is more efficient than C. ljungdahlii's (Islam et al., 2015; Nagarajan et al., 2013). We also observed increased ATP production by M. thermoacetica as compared to C. ljungdahlii during the simulation of MEG-production from $\mathrm{CO}$ and $\mathrm{CO}_{2}+\mathrm{H}_{2}$ using the 9 MEG-producing pathways in the organisms' in silico models. Hence, $M$. thermoacetica is possibly a better host for the metabolic engineering of MEG-producing pathways. Overall, MEG2, MEG5, and MEG7 showed the best performance in terms of pathway yields estimation using both $M$. thermoacetica and C. ljungdahlii models (Figures 8A and 8B).

\section{Conclusion}

Utilizing the power of cheminformatics tool and the promise of natural carbon fixing pathways of acetogenic bacteria, we designed hundreds of novel, biologically unknown MEG-producing pathways from Acetyl-CoA and 7 previously unknown biological pathways to MEG from syngas. The novel pathways provide valuable opportunities for hypothesis generation and testing regarding their enzyme candidates, and the biological pathways open up new avenues for the production of MEG from gases. The pathway curation and pruning criteria developed and applied in this study can be used as a practical guide for designing novel biochemical pathways to myriad value-added commodity chemicals in order to ultimately achieve the metabolic engineering of those pathways to appropriate chassis organisms. Also, the application of genome-wide metabolic models of host organisms for in-depth analyses of pathway yields highlighted the importance of such detailed mathematical models in assessing and identifying superior pathways and better host microbes for the ultimate metabolic engineering purposes. 
Finally, the biological production of MEG from gaseous substrates, if realized experimentally by implementing the pathways proposed here in $M$. thermoacetica, will be an attractive and effective alternative to current fossil fuel-based unsustainable MEG-production methods.

\section{Acknowledgements}

MAI and GS would like to thank the SABIC Americas, Inc. for funding the project. VH, NH and MA acknowledge the Swiss National Science Foundation (SNF) and SystemsX.ch, the Swiss Initiative in Systems Biology for funding the project. 


\section{References}

Alkim, C., Cam, Y., Trichez, D., Auriol, C., Spina, L., Vax, A., Bartolo, F., Besse, P., François, J. M., Walther, T., 2015. Optimization of ethylene glycol production from (d)-xylose via a synthetic pathway implemented in Escherichia coli. Microbial cell factories. 14, 1-12.

Anwar, Z., Gulfraz, M., Irshad, M., 2014. Agro-industrial lignocellulosic biomass a key to unlock the future bio-energy: A brief review. Journal of Radiation Research and Applied Sciences. 7, 163-173.

Becker, S. A., Feist, A. M., Mo, M. L., Hannum, G., Palsson, B. O., Herrgard, M. J., 2007. Quantitative prediction of cellular metabolism with constraint-based models: the COBRA Toolbox. Nature Protocols. 2, 727-738.

Blanch, H. W., Clark, D. S., 1997. Stoichiometry and energetics of microbial growth. Biochemical Engineering. Marcel Dekker, Inc., New York, pp. 162-181.

Campodonico, M. A., Andrews, B. A., Asenjo, J. A., Palsson, B. O., Feist, A. M., 2014. Generation of an atlas for commodity chemical production in Escherichia coli and a novel pathway prediction algorithm, GEM-Path. Metabolic Engineering. 25, 140-158.

Carbonell, P., Parutto, P., Herisson, J., Pandit, S. B., Faulon, J. L., 2014. XTMS: Pathway design in an eXTended metabolic space. Nucleic Acids Research. 42, W389-W394.

Carbonell, P., Planson, A.-G., Faulon, J.-L., 2013. Retrosynthetic Design of Heterologous Pathways. In: Alper, S. H., (Ed.), Systems Metabolic Engineering: Methods and Protocols. Humana Press, Totowa, NJ, pp. 149-173.

Caspi, R., Altman, T., Billington, R., Dreher, K., Foerster, H., Fulcher, C. A., Holland, T. A., Keseler, I. M., Kothari, A., Kubo, A., Krummenacker, M., Latendresse, M., Mueller, L. A., Ong, Q., Paley, S., Subhraveti, P., Weaver, D. S., Weerasinghe, D., Zhang, P., Karp, P. D., 2014. The MetaCyc database of metabolic pathways and enzymes and the BioCyc collection of Pathway/Genome Databases. Nucleic Acids Research. 42, D459-D471.

Drake, H. L., 1994. Acetogenesis, Acetogenic Bacteria, and the Acetyl-CoA "Wood/Ljungdahl" Pathway: Past and Current Perspectives. In: Drake, H. L., (Ed.), Acetogenesis. Springer US, Boston, MA, pp. 3-60.

Drake, H. L., Gossner, A. S., Daniel, S. L., 2008. Old acetogens, new light. Annals of the New York Academy of Sciences. 1125, 100-28.

Dugar, D., Stephanopoulos, G., 2011. Relative potential of biosynthetic pathways for biofuels and bio-based products. Nat Biotech. 29, 1074-1078.

Fast, A. G., Papoutsakis, E. T., 2012. Stoichiometric and energetic analyses of nonphotosynthetic CO2-fixation pathways to support synthetic biology strategies for production of fuels and chemicals. Current Opinion in Chemical Engineering. 1, 380-395.

Fehér, T., Planson, A. G., Carbonell, P., Fernández-Castané, A., Grigoras, I., Dariy, E., Perret, A., Faulon, J. L., 2014. Validation of RetroPath, a computer-aided design tool for metabolic pathway engineering. Biotechnology Journal. 9, 1446-1457.

Finley, S. D., Broadbelt, L. J., Hatzimanikatis, V., 2009. Computational framework for predictive biodegradation. Biotechnology and Bioengineering. 104, 1086-1097.

Forkner, M. W., Robson, J. H., Snellings, W. M., Martin, A. E., Murphy, F. H., Parsons, T. E., 2000. Glycols. Kirk-Othmer Encyclopedia of Chemical Technology. John Wiley \& Sons, Inc. 
Fuchs, G., 2011. Alternative pathways of carbon dioxide fixation: insights into the early evolution of life? Annual review of microbiology. 65, 631-58.

GrandViewResearchReport, http://www.grandviewresearch.com/industry-analysis/ethyleneglycols-industry. 2016.

Gupta, N. K., Vennesland, B., 1964. Glyoxylate carboligase of Escherichia coli: a flavoprotein. J Biol Chem. 239, 3787-9.

Hadadi, N., Hafner, J., Shajkofci, A., Zisaki, A., Hatzimanikatis, V., 2016. ATLAS of Biochemistry: A Repository of All Possible Biochemical Reactions for Synthetic Biology and Metabolic Engineering Studies. ACS Synthetic Biology.

Hadadi, N., Hatzimanikatis, V., 2015. Design of computational retrobiosynthesis tools for the design of de novo synthetic pathways. Current Opinion in Chemical Biology. 28, 99-104.

Hatzimanikatis, V., Li, C., Ionita, J. A., Henry, C. S., Jankowski, M. D., Broadbelt, L. J., 2005. Exploring the diversity of complex metabolic networks. Bioinformatics. 21, 1603-1609.

Huang, H., Wang, S., Moll, J., Thauer, R. K., 2012. Electron bifurcation involved in the energy metabolism of the acetogenic bacterium Moorella thermoacetica growing on glucose or H2 plus CO2. Journal of bacteriology. 194, 3689-99.

Hyduke, D., Schellenberger, J., Que, R., Fleming, R., Thiele, I., Orth, J., Feist, A., Zielinski, D., Bordbar, A., Lewis, N., Rahmanian, S., Kang, J., Palsson, B., 2011. COBRA Toolbox 2.0. Protocol Exchange.

Islam, M. A., Zengler, K., Edwards, E. A., Mahadevan, R., Stephanopoulos, G., 2015. Investigating Moorella thermoacetica metabolism with a genome-scale constraint-based metabolic model. Integrative biology : quantitative biosciences from nano to macro. 7 , 869-82.

Jankowski, M. D., Henry, C. S., Broadbelt, L. J., Hatzimanikatis, V., 2008. Group contribution method for thermodynamic analysis of complex metabolic networks. Biophysical Journal. 95, 1487-1499.

Kanehisa, M., Sato, Y., Kawashima, M., Furumichi, M., Tanabe, M., 2016. KEGG as a reference resource for gene and protein annotation. Nucleic Acids Research. 44, D457-D462.

Kim, S., Thiessen, P. A., Bolton, E. E., Chen, J., Fu, G., Gindulyte, A., Han, L., He, J., He, S., Shoemaker, B. A., Wang, J., Yu, B., Zhang, J., Bryant, S. H., 2016. PubChem Substance and Compound databases. Nucleic Acids Research. 44, D1202-D1213.

Kopke, M., Held, C., Hujer, S., Liesegang, H., Wiezer, A., Wollherr, A., Ehrenreich, A., Liebl, W., Gottschalk, G., Durre, P., 2010. Clostridium ljungdahlii represents a microbial production platform based on syngas. Proceedings of the National Academy of Sciences of the United States of America. 107, 13087-92.

Li, C., Henry, C. S., Jankowski, M. D., Ionita, J. A., Hatzimanikatis, V., Broadbelt, L. J., 2004. Computational discovery of biochemical routes to specialty chemicals. Chemical Engineering Science. 59, 5051-5060.

Liu, H., Ramos, K. R., Valdehuesa, K. N., Nisola, G. M., Lee, W. K., Chung, W. J., 2013. Biosynthesis of ethylene glycol in Escherichia coli. Applied microbiology and biotechnology. 97, 3409-17.

Mackaluso, J. D., 2007. The use of syngas derived from biomass and waste products to produce ethanol and hydrogen. MMG445 Basic Biotechnology eJournal. 3, 98-103.

Medema, M. H., van Raaphorst, R., Takano, E., Breitling, R., 2012. Computational tools for the synthetic design of biochemical pathways. Nat Rev Micro. 10, 191-202. 
Menon, V., Rao, M., 2012. Trends in bioconversion of lignocellulose: Biofuels, platform chemicals \&amp; biorefinery concept. Progress in Energy and Combustion Science. 38, $522-550$.

Mock, J., Wang, S., Huang, H., Kahnt, J., Thauer, R. K., 2014. Evidence for a hexaheteromeric methylenetetrahydrofolate reductase in Moorella thermoacetica. Journal of bacteriology. 196, 3303-14.

Nagarajan, H., Sahin, M., Nogales, J., Latif, H., Lovley, D. R., Ebrahim, A., Zengler, K., 2013. Characterizing acetogenic metabolism using a genome-scale metabolic reconstruction of Clostridium ljungdahlii. Microbial cell factories. 12, 118.

NC-IUBMB, 1992. Nomenclature Committee of the International Union of Biochemistry and Molecular Biology. http://www.chem.qmul.ac.uk/iubmb/enzyme/.

Orth, J. D., Thiele, I., Palsson, B. Ø., 2010. What is flux balance analysis? Nature biotechnology. 28, 245-248.

Pereira, B., Li, Z. J., De Mey, M., Lim, C. G., Zhang, H., Hoeltgen, C., Stephanopoulos, G., 2016a. Efficient utilization of pentoses for bioproduction of the renewable two-carbon compounds ethylene glycol and glycolate. Metab Eng. 34, 80-7.

Pereira, B., Zhang, H., De Mey, M., Lim, C. G., Li, Z. J., Stephanopoulos, G., $2016 b$. Engineering a novel biosynthetic pathway in Escherichia coli for production of renewable ethylene glycol. Biotechnol Bioeng. 113, 376-83.

Pierce, E., Xie, G., Barabote, R. D., Saunders, E., Han, C. S., Detter, J. C., Richardson, P., Brettin, T. S., Das, A., Ljungdahl, L. G., Ragsdale, S. W., 2008. The complete genome sequence of Moorella thermoacetica (f. Clostridium thermoaceticum). Environmental microbiology. 10, 2550-73.

Rebsdat, S., Mayer, D., 2000. Ethylene Glycol. Ullmann's Encyclopedia of Industrial Chemistry. Wiley-VCH Verlag GmbH \& Co. KGaA.

Rodrigo, G., Carrera, J., Prather, K. J., Jaramillo, A., 2008. DESHARKY: Automatic design of metabolic pathways for optimal cell growth. Bioinformatics. 24, 2554-2556.

Rostrup-Nielsen, J., Christiansen, L. J., 2011. Concepts in syngas manufacture. Imperial College Press.

Schuchmann, K., Muller, V., 2014. Autotrophy at the thermodynamic limit of life: a model for energy conservation in acetogenic bacteria. Nature reviews. Microbiology. 12, 809-21.

Shearer, A. G., Altman, T., Rhee, C. D., 2014. Finding Sequences for over 270 Orphan Enzymes. PLoS ONE. 9, e97250.

ShellFactsSheet, http://www.shell.com/business-customers/chemicals/factsheets-speeches-andarticles/factsheets/mono-ethylene-glycol.html. Vol. 2016, 2016.

Sorokina, M., Stam, M., Médigue, C., Lespinet, O., Vallenet, D., 2014. Profiling the orphan enzymes. Biology Direct. 9, 1-16.

Stephanopoulos, G., Pereira, B., De, M. M., Dugar, D., Avalos, J. L., Engineering microbes and metabolic pathways for the production of ethylene glycol. Vol. Patent. WO2013126721 A1, 2013.

Wang, S., Huang, H., Kahnt, J., Thauer, R. K., 2013. A reversible electron-bifurcating ferredoxin- and NAD-dependent [FeFe]-hydrogenase (HydABC) in Moorella thermoacetica. Journal of bacteriology. 195, 1267-75.

Watschinger, K., Werner, E. R., 2013. Orphan enzymes in ether lipid metabolism. Biochimie. 95, 59-65. 


\section{Figure legends}

Figure 1. BNICE.ch generated compounds and reactions for MEG-synthesis. The total number of compounds and reactions retrosynthetically generated by BNICE.ch are illustrated. Since KEGG was used as the reference database for running BNICE.ch to generate MEG from Acetyl-CoA, all compounds/metabolites in the reconstructed biochemical network belonged to KEGG as shown by the solid line; only $18 \%$ of the total generated reactions belonged to KEGG (represented by blue bars), while the remaining $82 \%$ of the BNICE.ch generated reactions are novel reactions without any existence in KEGG (see text for details).

Figure 2. Different categories of the MEG-producing pathways. The MEG-producing pathways connecting Acetyl-CoA to MEG were divided into different categories based on the presence of common metabolite pairs in the last step/reaction of a pathway i.e., by applying the $2^{\text {nd }}$ pathway pruning criterion to all BNICE.ch generated pathways (see text for details). The categories of 3-step and 4-step pathways are shown in (A) and (B), respectively. The accepted pathways are the ones that passed the $3^{\text {rd }}$ and $4^{\text {th }}$ pathway pruning criteria, and the discarded pathways failed to pass those pruning criteria (see text for details).

Figure 3. Overview of KEGG-reactions in the pruned MEG-producing pathways. The total number of KEGG-reactions i.e., biologically known reactions present in different categories of accepted/pruned 3-step and 4-step pathways is shown in (A) and (B), respectively. The EthanolMEG and Glycolaldehyde-MEG categories of 3-step pathways include the highest number (4 and 2, respectively) of most KEGG-reaction containing (2 KEGG-steps) pathways as shown in 
A. The same categories of 4-step pathways contain the highest number (12 and 12, respectively) of most KEGG-reaction containing (3 KEGG-steps) pathways as shown in B. The pathways in the Glycerate-MEG category include at most 1 KEGG-reaction for the 3-step pathways (A) and 2 KEGG-reactions for the 4-step pathways (B).

Figure 4. Biochemical network analysis of the pruned MEG-producing pathways. The reconstructed biochemical networks of the pruned MEG-producing pathways of 3 and 4-step length are schematically illustrated in the figure: Ethanol-MEG, Glycerate-MEG, and Glycolaldehyde-MEG categories of 3-step long pruned pathways are shown in (A), (B), and (C), respectively; (D), (E), and (F) are showing similar categories of 4-step pathways. The categories are generated from the implementation of the $2^{\text {nd }}$ pruning criterion towards the BNICE.ch generated pathways (see text for details). The nodes and edges in a network are representing the metabolites and reactions, respectively, and the KEGG-reaction number (R\#\#\#\#) corresponding to an edge indicates if that reaction belongs to the KEGG database.

\section{Figure 5. Model-based pathway yield analysis of the pruned MEG-producing pathways.}

The pathway MEG-yields of all 3 and 4-step long, pruned and accepted pathways estimated using the published $M$. thermoacetica metabolic model (Islam et al., 2015) growing on $\mathrm{H}_{2}+\mathrm{CO}_{2}$ and $\mathrm{CO}$ are shown in (A) and (B), respectively. The MEG-yields of the same pathways on the same substrates estimated using the published C. ljungdahlii metabolic model (Nagarajan et al., 2013) are shown in (C) and (D). Some pathways generated zero MEG-yields with the $C$. ljungdahlii model due to the incompatibility of the pathways with the host metabolism (see text 
for details). The bubble sizes are corresponding to the number of pathways generating a specific pathway yield.

Figure 6. Schematic overview of the MEG-producing pathways containing only KEGGreactions. The MEG-producing pathways containing only KEGG-reactions i.e., biologically known reactions from Glycerate (MEG1 - MEG4) are schematically shown in (A) and from Ethanol (MEG5 - MEG7) in (B). The nodes and edges are representing the metabolites and reactions, respectively, and the KEGG-reaction numbers (R\#\#\#\#) corresponding to an edge indicate if those reactions belong to the KEGG database. Multiple KEGG-reaction numbers corresponding to some edges/arrows indicate the availability of multiple reactions for the respective conversions of metabolites in a pathway. The overall pathway from syngas to MEG is shown in (C) by incorporating both previously known (the Wood-Ljungdahl pathway, gluconeogenesis, and Ethanol fermentation) and the newly identified (MEG1 - MEG7) biological pathways.

Figure 7. Thermodynamic feasibility and gene availability analyses of the MEG-producing pathways containing only KEGG-reactions. (A) shows the comparison of the overall pathway Gibbs energy changes of the newly identified 7 pathways with the 2 published pathways from xylose (Liu et al., 2013) and glucose (Pereira et al., 2016b). The pathway Gibbs energy changes were estimated from the standard Gibbs energy changes of the reactions comprising a pathway (see text for details). (B) shows the comparative gene availability analysis of the same pathways. If the gene(s) associated with the corresponding steps/reactions in a pathway are present in the hosts $M$. thermoacetica (MT) and C. ljungdahlii (CL), they are represented by the green color. If 
the gene(s) are found in other sequenced genomes in the KEGG database, they are represented by the yellow color. Black represents the gene(s) that is not found in any sequenced genome i.e., the orphan enzymes.

Figure 8. Model-based pathway yield analysis of the MEG-producing pathways containing only KEGG-reactions. (A) shows the pathway yield analysis of the newly identified 7 pathways and the 2 published pathways from xylose (Liu et al., 2013) and glucose (Pereira et al., 2016b) using $\mathrm{H}_{2}+\mathrm{CO}_{2}$ as substrate. (B) shows the same analysis for the same pathways using $\mathrm{CO}$ as substrate. In both cases, the MEG-yield was estimated by inserting the pathways into the published genome-scale models of $M$. thermoacetica (MT) (Islam et al., 2015) and C. ljungdahlii (CL) (Nagarajan et al., 2013), and running model simulations for each pathway using $\mathrm{H}_{2}+\mathrm{CO}_{2}$ and $\mathrm{CO}$ as substrates (see text for details). 


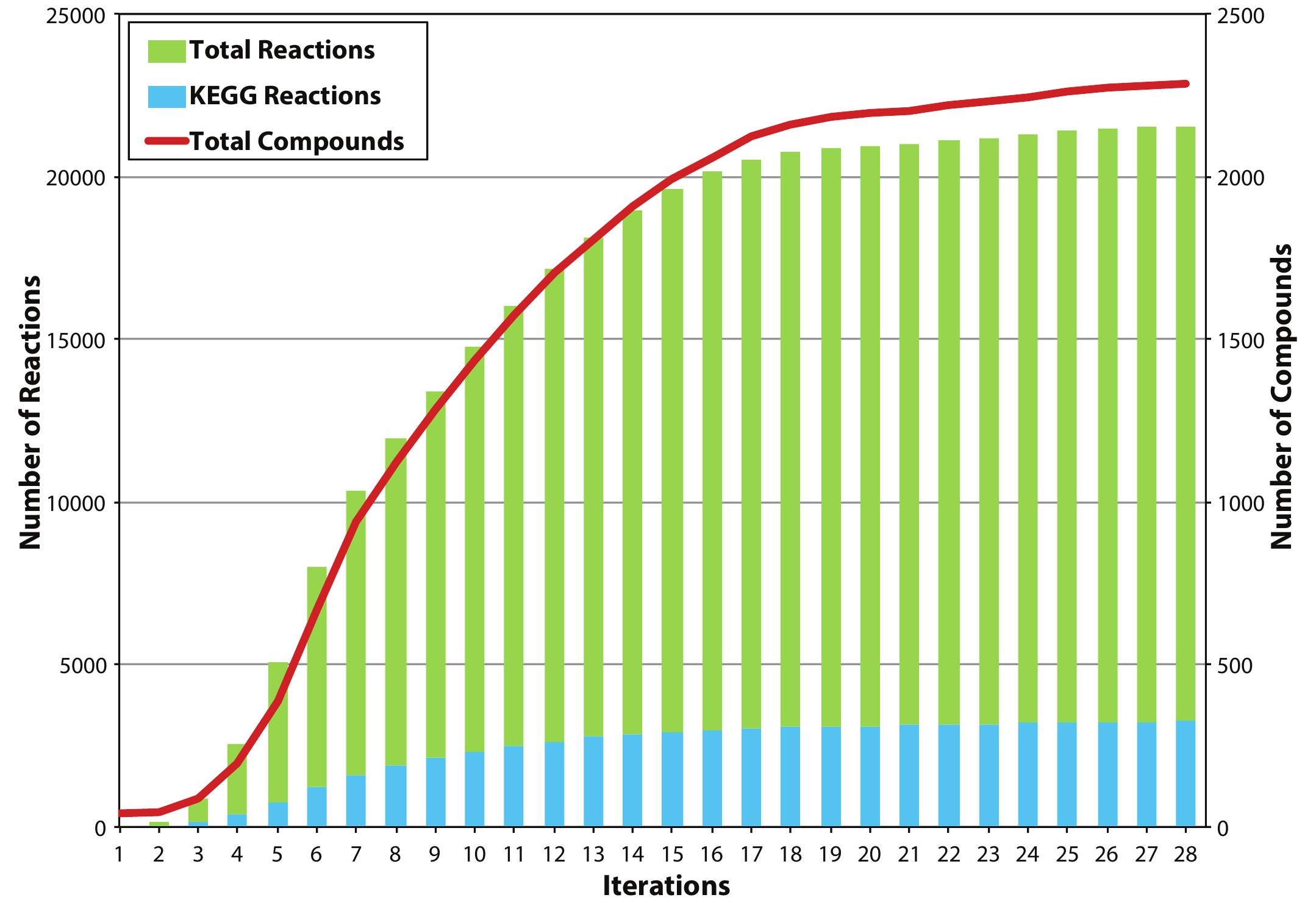




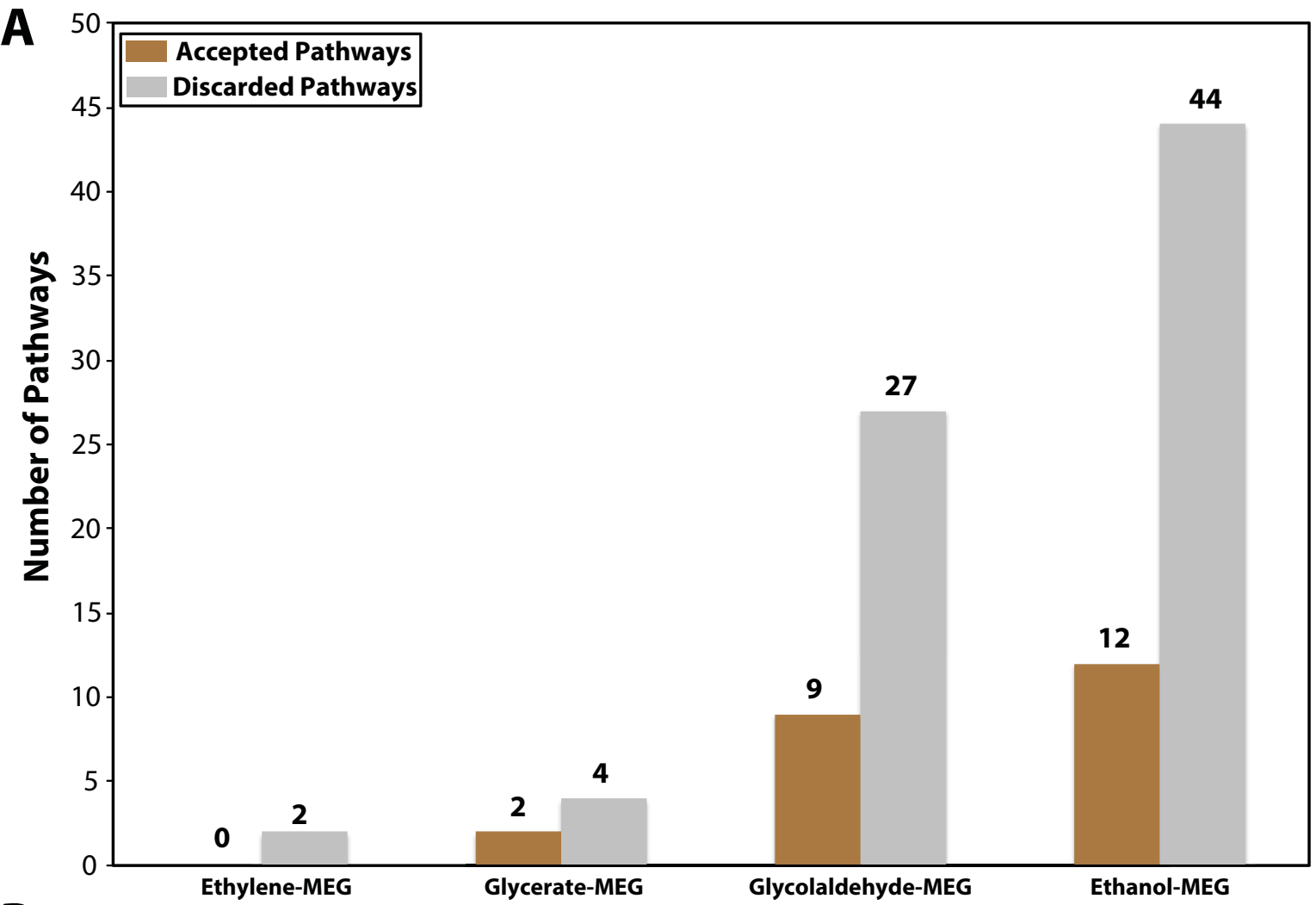

B

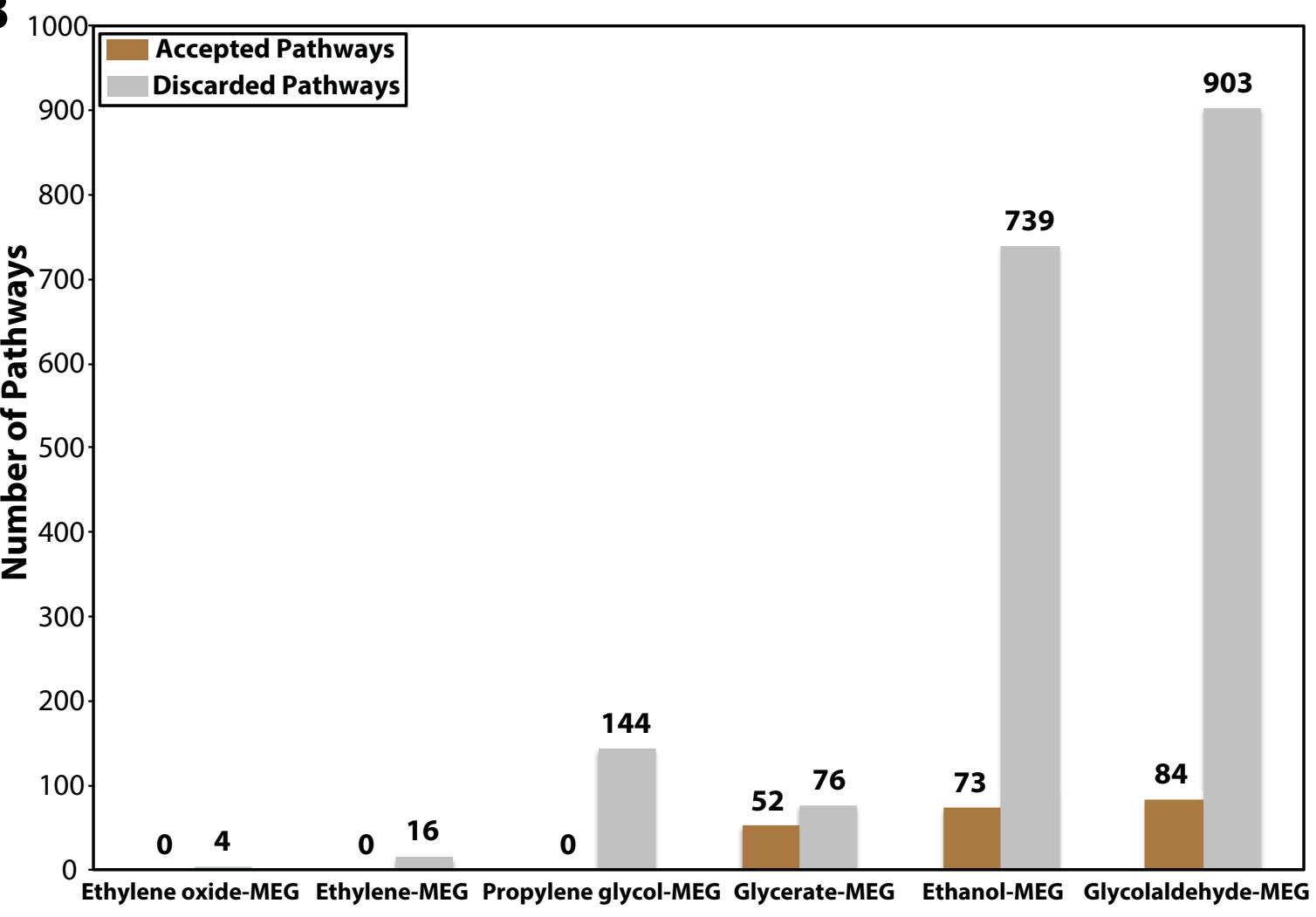




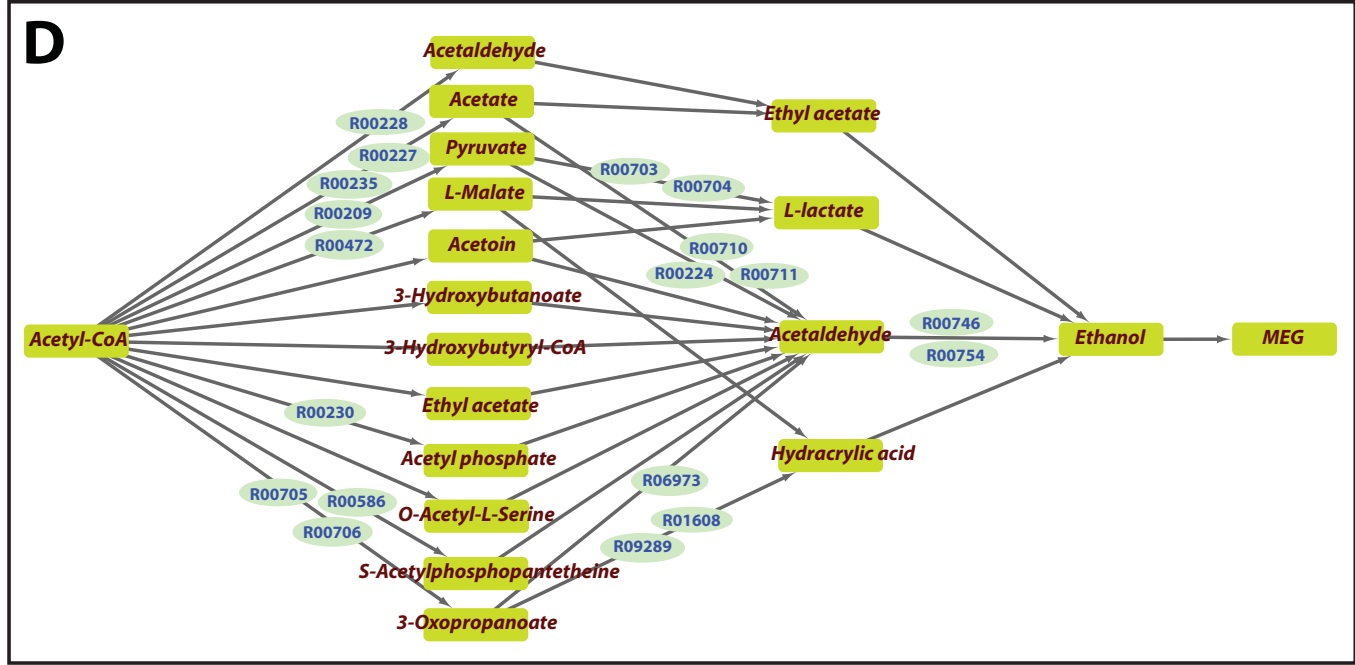

\section{B}

Acetyl-CoA $\stackrel{\text { R00209 }}{\longrightarrow}$ Pyruvate $\longrightarrow$ Glycerate $\longrightarrow$ MEG

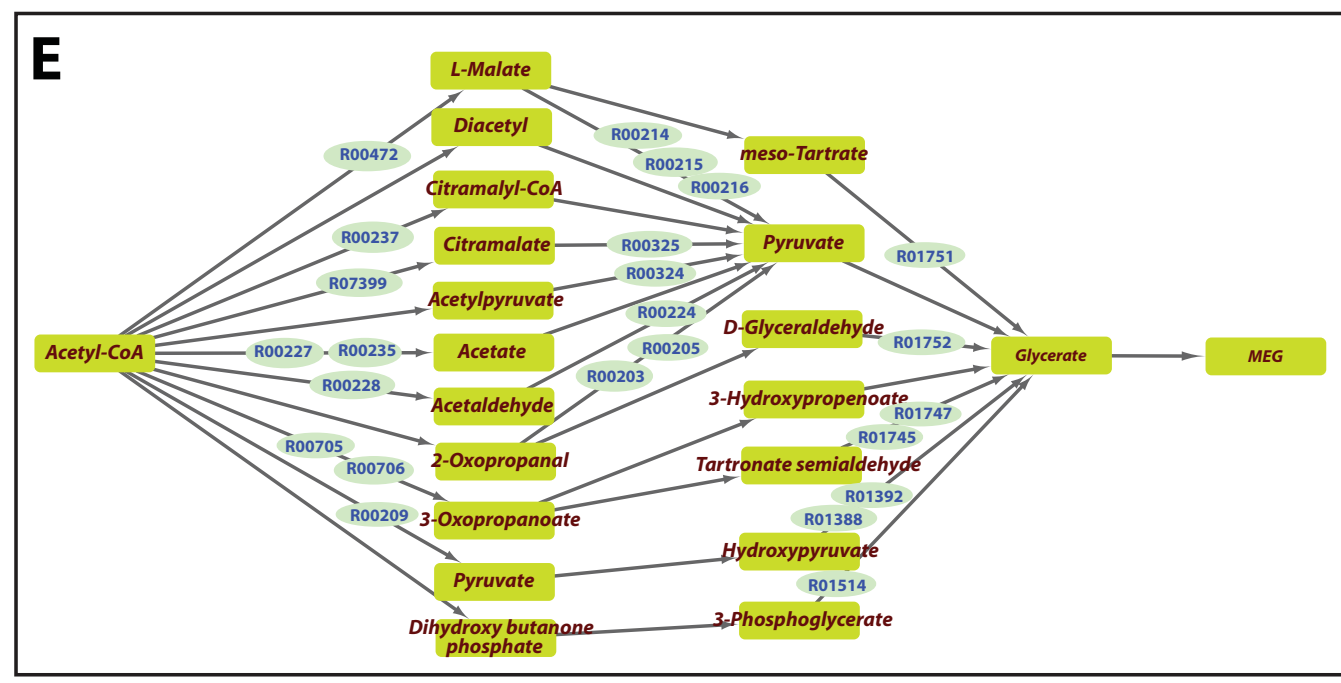

F

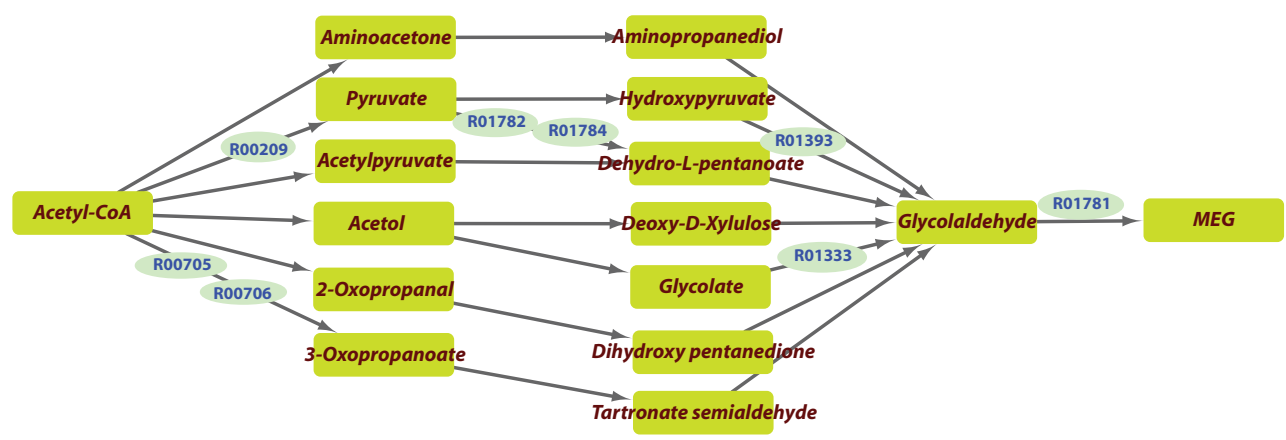

Acetyl-CoA $\stackrel{\text { R00228 }}{\longrightarrow}$ Acetaldehyde $\longrightarrow$ Glycolaldehyde $\stackrel{\text { R01781 }}{\longrightarrow}$ MEG


B
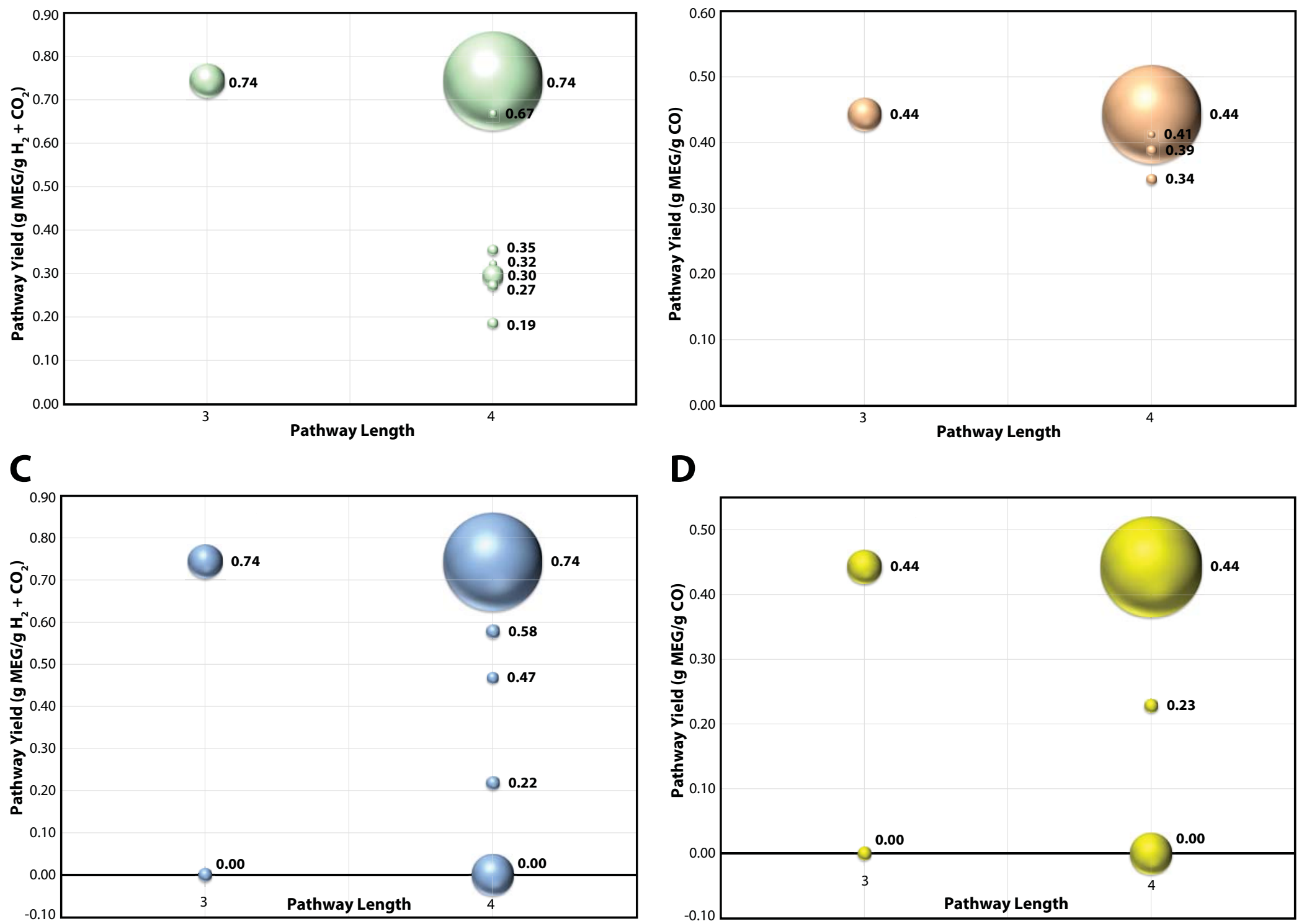


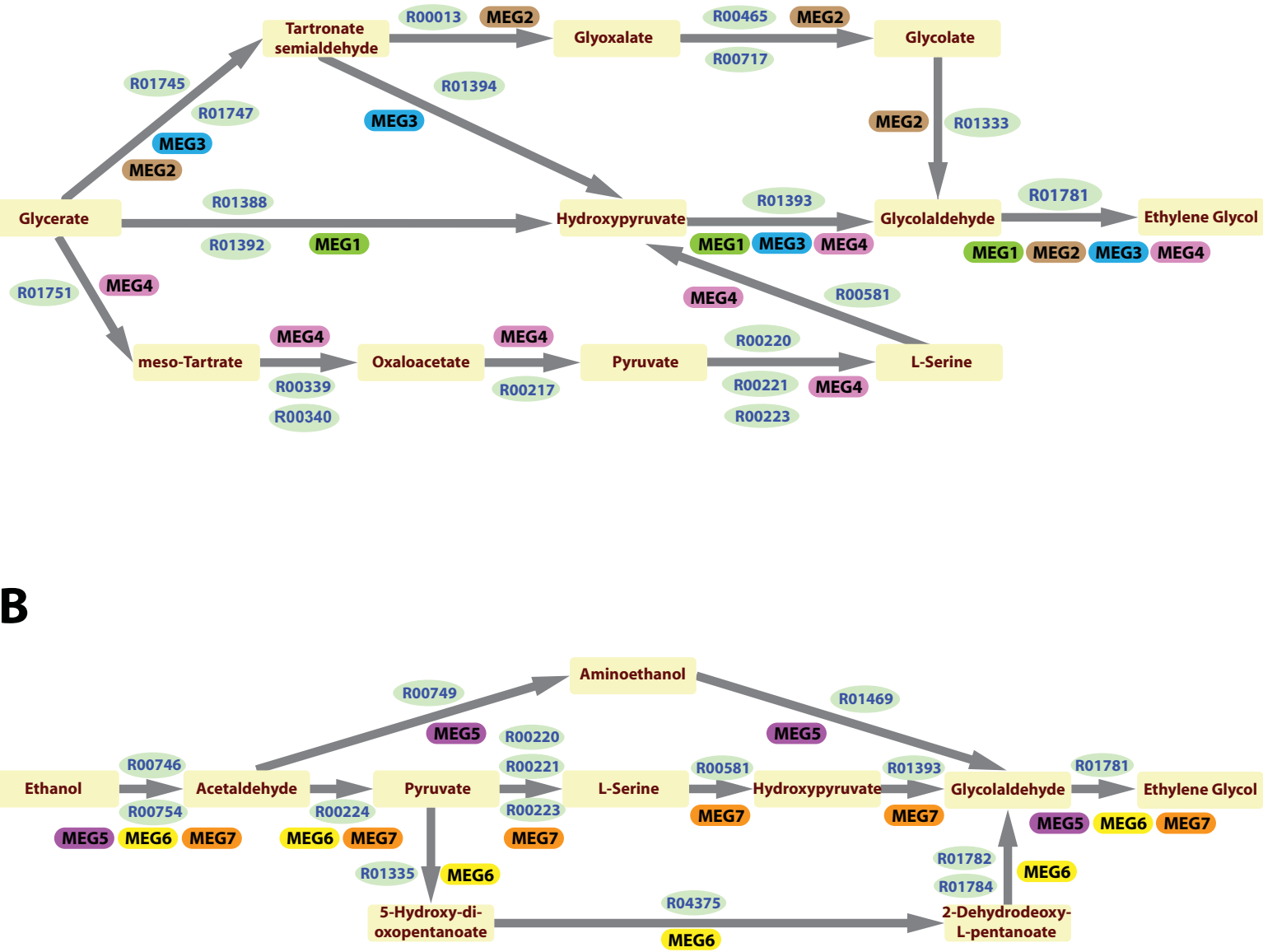

\section{Syngas}




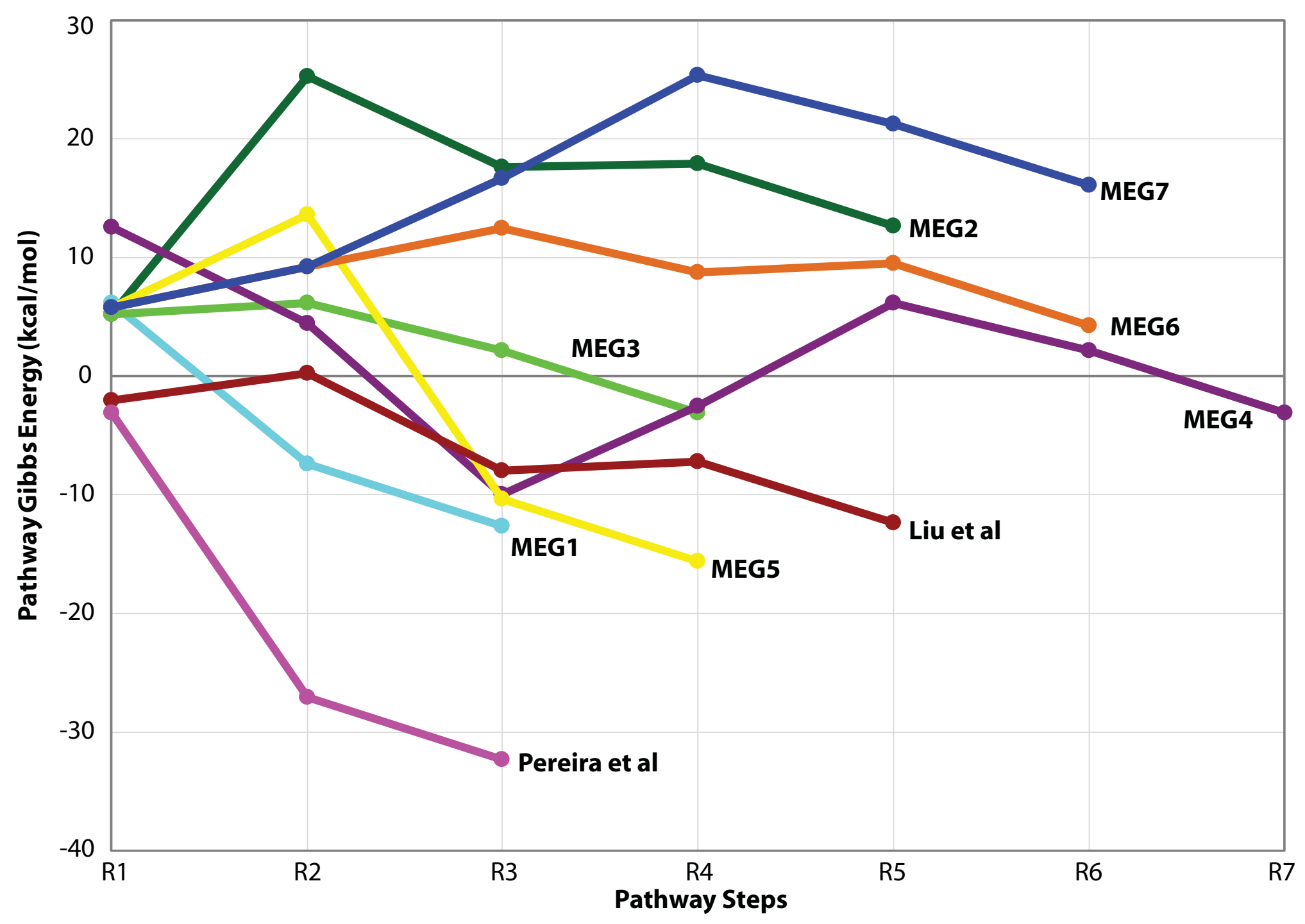

\begin{tabular}{|c|c|c|c|c|c|c|c|c|}
\hline $\begin{array}{r}\text { Steps } \rightarrow \\
\text { Pathways } \\
\downarrow\end{array}$ & $\mathrm{R} 1$ & $\mathrm{R} 2$ & R3 & R4 & R5 & R6 & R7 & \\
\hline MEG1 & & & & & & & & MT/CL \\
\hline MEG2 & & & & & & & & Other \\
\hline MEG3 & & & & & & & & Orphan \\
\hline MEG4 & & & & & & & & \\
\hline MEG5 & & & & & & & & \\
\hline MEG6 & & & & & & & & \\
\hline MEG7 & & & & & & & & \\
\hline Liu et al & & & & & & & & \\
\hline Pereira et a & & & & & & & & \\
\hline
\end{tabular}



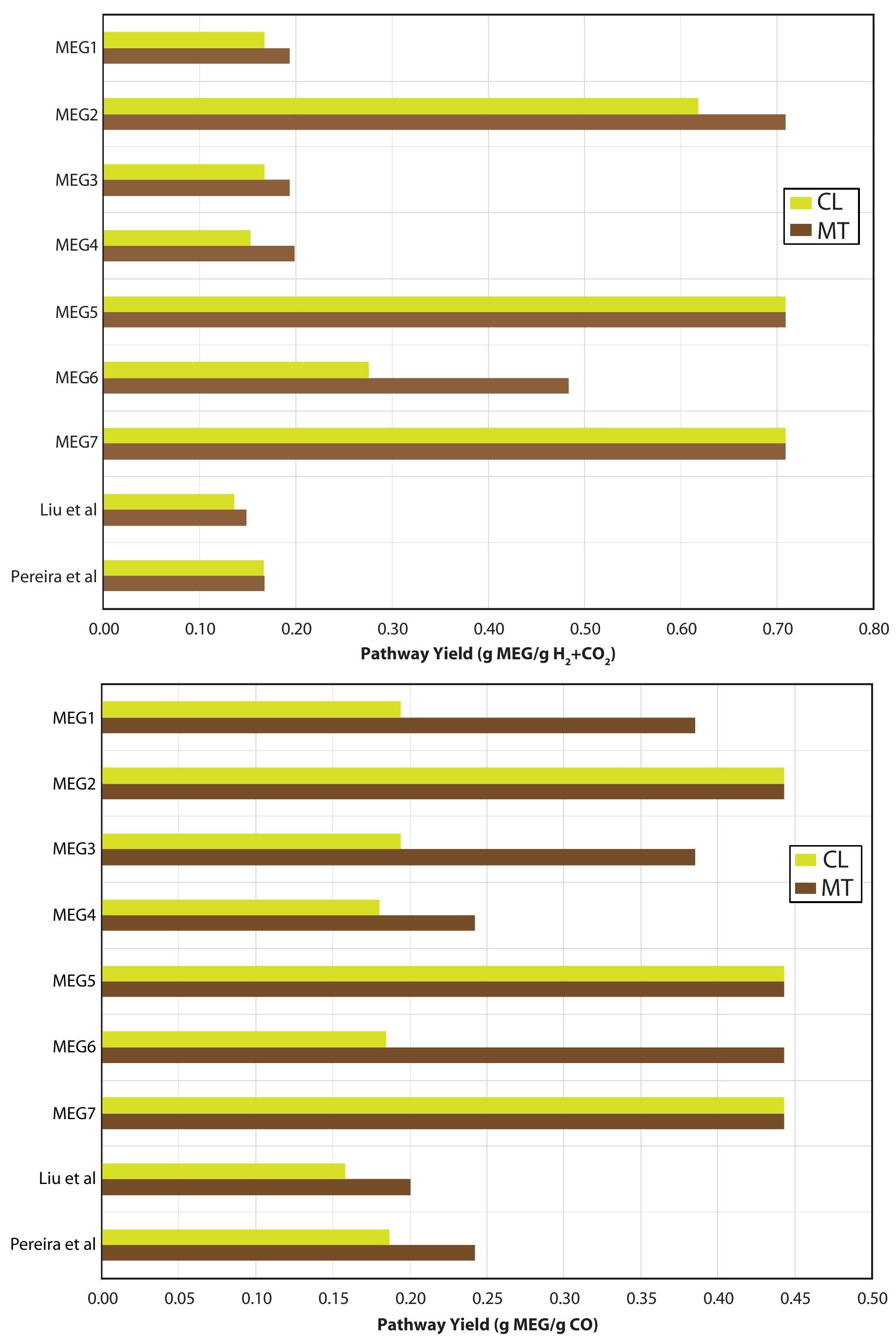